\title{
RAVSKY A.
}

\section{A NOTE ON COMPACT-LIKE SEMITOPOLOGICAL GROUPS}

\begin{abstract}
We present a few results related to separation axioms and automatic continuity of operations in compact-like semitopological groups. In particular, is provided a semiregular semitopological group $G$ which is not $T_{3}$. We show that each weakly semiregular compact semitopological group is a topological group. On the other hand, constructed examples of quasiregular $T_{1}$ compact and $T_{2}$ sequentially compact quasitopological groups, which are not paratopological groups. Also we prove that a semitopological group $(G, \tau)$ is a topological group provided there exists a Hausdorff topology $\sigma \supset \tau$ on $G$ such that $(G, \sigma)$ is a precompact topological group and $(G, \tau)$ is weakly semiregular or $(G, \sigma)$ is a feebly compact paratopological group and $(G, \tau)$ is $T_{3}$.

Key words and phrases: semitopological group, paratopological group, compact-like semitopological group, compact-like paratopological group, continuity of the inverse, joint continuity, separation axioms, countably compact paratopological group, feebly compact topological group, countably compact topological group.
\end{abstract}

Pidstryhach Institute for Applied Problems of Mechanics and Mathematics, 3b Naukova str., 79060, Lviv, Ukraine E-mail: alexander.ravsky@uni-wuerzburg.de

\section{PRELIMINARIES}

In this paper the word "space" means "topological space".

\subsection{Topologized groups}

A topologized group $(G, \tau)$ is a group $G$ endowed with a topology $\tau$. It is called a semitopological group provided the multiplication map $G \times G \rightarrow G,(x, y) \mapsto x y$ is separately continuous. Moreover, if the multiplication is continuous then $G$ is called a paratopological group. A semitopological group with the continuous inversion map $G \rightarrow G, x \mapsto x^{-1}$ is called a quasitopological group. A topologized group which is both paratopological and quasitopological is called a topological group.

Whereas investigation of topological groups already is one of fundamental branches of topological algebra (see, for instance, [11,29] and [5]), other topologized groups are not so well-investigated and have more variable structure.

Basic properties of semitopological or paratopological groups are described in book [5] by Arhangel'skii and Tkachenko, in author's PhD thesis [32] and papers [30,31]. New Tkachenko's survey [40] presents recent advances in this area.

$\overline{\mathrm{y} \triangle \mathrm{K} 512.546 .82,512.546 .8,512.546}$

2010 Mathematics Subject Classification: 22A15, 54H99, 54H11.

This work was supported by the budget program of Ukraine "Support for the development of priority research areas" (CPCEC 6451230). 


\subsection{Separation axioms}

These axioms describe specific structural properties of a space. Basic separation axioms and relations between them are considered in [16, Section 1.5]. For more specific cases and topics, also related to semitopological and paratopological groups, see [7,31], [40, Section 2], [22,41].

All spaces considered in the present paper are not supposed to satisfy any of the separation axioms, if otherwise is not stated. We recall separation axioms which we use in our paper. A space $X$ is

- $T_{0}$, if for any distinct points $x, y \in X$ there exists an open set $U \subset X$, which contains exactly one of the points $x, y$,

- $T_{1}$, if for any distinct points $x, y \in X$ there exists an open set $x \in U \subset X \backslash\{y\}$,

- $T_{2}$ or Hausdorff, if any distinct points $x, y \in X$ have disjoint neighborhoods,

- $T_{3}$, if any closed set $F \subset X$ and any point $x \in X \backslash F$ have disjoint neighborhoods,

- regular, if it is $T_{1}$ and $T_{3}$,

- quasiregular, if any nonempty open subset $A$ of $X$ contains the closure of some nonempty open subset $B$ of $X$,

- weakly semiregular, if $X$ has a base consisting of regular open sets, that is such sets $U$ that $U=\operatorname{int} \bar{U}$,

- semiregular, if it is weakly semiregular and $T_{2}$,

- functionally $T_{2}$ of functionally Hausdorff, if for any distinct points $x, y \in X$ there exists a continuous function $f: X \rightarrow \mathbb{R}$ such that $f(x) \neq f(y)$,

- $T_{3 \frac{1}{2}}$ or completely regular, if it is $T_{1}$ and for any closed set $F \subset X$ and any point $x \in X \backslash F$ there exists a continuous function $f: X \rightarrow \mathbb{R}$ such that $f(x)=0$ and $f(F) \subset\{1\}$.

Remark that each $T_{3}$ space is quasiregular and weakly semiregular, so each regular space is semiregular.

\subsection{Separation axioms in semitopological groups}

It is easy to show that each topological group is $T_{3}$. Near 1936 Pontrjagin showed that each $T_{0}$ topological group is completely regular and $T_{1}$.

On the other hand, simple examples shows that for paratopological groups neither of the implications $T_{0} \Rightarrow T_{1} \Rightarrow T_{2} \Rightarrow T_{3}$ is necessary (see [30, Examples 1.6-1.8] and page 5 in any of papers [31] or [40]) and there are only a few backwards implications between different separation axioms, see [31, Section 1] or [40, Section 2]. Moreover, in 2014 Banakh and the author of the present paper similarly to Pontrjagin's proof showed that each $T_{1}$ weakly semiregular paratopological group is $T_{3 \frac{1}{2}}$ and each $T_{2}$ paratopological group is functionally $T_{2}$ [7]. On the other hand, Banakh's announcement for a seminar for 28 November 2016 (see [39]) claims on an example of a regular quasitopological group which is not functionally Hausdorff. 
It is easy to show that each weakly semiregular paratopological group is $T_{3}$ [31, Proposition 1.5], but there exists a semiregular semitopological group $G$ which is not $T_{3}$, see Example 1 . On the other hand, in Proposition 1 we shall prove that each $T_{0}$ weakly semiregular semitopological group is semiregular.

Given a topological space $(X, \tau)$ Stone [38] and Katĕtov [18] considered the topology $\tau_{s r}$ on $X$ generated by the base consisting of all regular open sets of the space $(X, \tau)$. This topology is called the semiregularization of the topology $\tau$. If $(X, \tau)$ is a semitopological group then $\left(X, \tau_{s r}\right)$ is a weakly semiregular semitopological group (see [31, p. 96]). If $(X, \tau)$ is a paratopological group then $\left(X, \tau_{s r}\right)$ is a $T_{3}$ paratopological group [31, Ex. 1.9], [32, p. 31], and [32, p. 28].

\subsection{Compact-like spaces}

Different classes of compact-like spaces and relations between them provide a well-known investigation topic of general topology, see, for instance, basic [16, Chap. 3] and general [13, $23,25,37,42]$ works. The including relations between the classes are often visually represented by arrow diagrams, see, [25, Diag. 3 at p.17], [12, Diag. 1 at p. 58] (for completely regular spaces), [37, Diag. 3.6 at p. 611], and [17, Diag. at p. 3].

We recall the definitions of compact-like spaces with which we shall deal in the paper. A space $X$ is called

- sequentially compact, if each sequence of $X$ contains a convergent subsequence,

- countably compact, if each countable open over of $X$ has a finite subcover,

- feebly compact, if each locally finite family of nonempty open subsets of the space $X$ is finite,

- pseudocompact, if $X$ is $T_{1}$ completely regular and each continuous real-valued function on $X$ is bounded.

It is well-known and easy to show that each (sequentially) compact space is countable compact and each countable compact space is feebly compact. Moreover, by [16, Theorem 3.10.22] a $T_{1}$ completely regular space is feebly compact iff it is pseudocompact.

\subsection{Automatic continuity of operations in semitopological groups}

It turned out that if a space of a semitopological (resp. paratopological) group satisfies some conditions (sometimes with some conditions imposed on the group) then the multiplication (resp. inversion) in the group is continuous, that is the group is topological (resp. paratopological). Investigation of these conditions is one of main branches of the theory of paratopological groups, and, as far as the author knows, the firstly developed that. It turned out that automatic continuity essentially depends on compact-like properties and separation axioms of the space of a semitopological group. An interested reader can find known results and references on this subject in the survey Section 5.1 of [32] and in Section 3 of the survey [40] (both for semitopological and paratopological groups), and in Introduction of [1], [8, Section 1.6](for paratopological groups).

We briefly recall the history of the topic. In 1936 Montgomery [26] showed that every completely metrizable paratopological group is a topological group. In 1953 Wallace [43] asked whether every locally compact regular semitopological group a topological group. In 1957 Ellis 
obtained a positive answer of the Wallace question (see [14,15]) (remark that later the author of the present paper showed that regularity condition can be relaxed, see Proposition 5.5 in [32] or its counterpart in English in [33]). In 1960 Zelazko used Montgomery's result and showed that each completely metrizable semitopological group is a topological group. Since both locally compact and completely metrizable topological spaces are Čech-complete (recall that Čechcomplete spaces are $G_{\delta}$-subspaces of Hausdorff compact spaces), this suggested Pfister [28] in 1985 to ask whether each Cech-complete semitopological group a topological group. In 1996 Bouziad [9] and Reznichenko [36], as far as the author knows, independently answered affirmatively to the Pfister's question. To do this, it was sufficient to show that each Čech-complete semitopological group is a paratopological group since earlier, Brand [10] had proved that every Cech-complete paratopological group is a topological group. Brand's proof was later improved and simplified in [28]. For recent advances in this topic see Moors' paper [27] and references there.

If $G$ is a paratopological group which is a $T_{1}$ space and $G \times G$ is countably compact (in particular, if $G$ is sequentially compact) then $G$ is a topological group, see [34]. On the other hand, we cannot weaken $T_{1}$ to $T_{0}$ here, because there exists a sequentially compact $T_{0}$ paratopological group which is not a topological group, see Example 5.27 from [8]. Also we cannot weaken countable compactness of $G \times G$ to that of $G$ because under additional axiomatic assumptions there exists a countably compact (free abelian) paratopological group which is not a topological group, see [8, Example 3.22]. Also there exists a functionally Hausdorff second countable feebly compact paratopological group $G$ which is not a topological group, see [8, Example 3.30]. On the other hand, by Proposition 3.15 from [8] each feebly compact quasiregular paratopological group is a topological group. In particular, each pseudocompact paratopological group is a topological group, see also [4, Theorem 1.7] and [2, Theorem 2.1].

According to [24, Corollary 6.3], a subgroup of a compact Hausdorff semitopological semigroup is a topological group. On the other hand, The group of integers $(\mathbb{Z},+)$ endowed with the cofinite topology is a $T_{1}$ compact semitopological group which is not a paratopological group. On the other hand, it is easy to check that each $T_{1}$ regular countably compact space is strongly Baire (see, [19, p.158] for definition), so by [19, Theorem 2], each $T_{1}$ regular countably compact semitopological group $G$ is a topological group. Nevertheless, there exists a pseudocompact quasitopological group $G$ of period 2, which is not a paratopological group, (see [20,21] and also [5, p.124-127]). On the other hand, Reznichenko in [35, Theorem 2.5] showed that each semitopological group $G \in \mathcal{N}$ is a topological group, where $\mathcal{N}$ is a family of all pseudocompact spaces $X$ such that $(X, X)$ is a Grothendieck pair, that is if each continuous image of $X$ in $C_{p}(Y)$ has the compact closure in $C_{p}(Y)$. In particular, a pseudocompact space $X$ belongs to $\mathcal{N}$ provided $X$ has one of the following properties: countable compactness, countable tightness, separability, $X$ is a $k$-space, see [35]. Also is known that every pseudocompact semitopological group of countable $\pi$-character is a compact metrizable topological group, see [5, Corollary 5.7.27]. Arhangel'skii, Choban, and Kenderov proved in [3, Proposition 8.5] that a $T_{2}$ locally countably compact semitopological group containing a compact of countable character is a paracompact locally compact topological group.

In the present paper we show that each weakly semiregular compact semitopological group $G$ is a topological group, see Theorem 1 . On the other hand, we construct examples of quasiregular $T_{1}$ compact and $T_{2}$ sequentially compact quasitopological groups, which are not paratopological groups, see Examples 2 and 3, respectively. 


\section{RESUlts}

Example 1. There exists a semiregular semitopological group $G$ which is not $T_{3}$. Put $G=$ $\left(\mathbb{R}^{2},+\right)$ and $\mathcal{B}=\left\{U_{n}: 0<n \in \mathbb{N}\right\}$, where $U_{n}=\{0\} \cup\left\{(x, y) \in \mathbb{R}^{2}:|y|<|x|<1 / n\right\}$ for each n. Put $\tau=\{V \subset G:(\forall x \in V)(\exists U \in \mathcal{B}): x+U \subset V\}$. It is easy to check that $(G, \tau)$ is a semitopological semigroup and $\mathcal{B}$ is its base at the unit. Let $\sigma$ be the standard topology of $\mathbb{R}^{2}$. Since $\tau \supset \sigma$, the group $(G, \tau)$ is $T_{2}$. Since int $\bar{U}^{\sigma}=U$ for each $U \in \mathcal{B}$, the group $(G, \tau)$ has a base $\{x+U: x \in G, U \in \mathcal{B}\}$, consisting of regular open sets. But the group $(G, \tau)$ is not $T_{3}$, because $U_{1} \not \supset{\overline{U_{n}}}^{\tau}$ for each $n$.

Let $G$ be a semitopological group and $H \subset G$ be a normal subgroup of $G$. It is easy to check that the quotient group $G / H$ endowed with the quotient topology with respect to the quotient map $\pi: G \rightarrow G / H$ is a semitopological group.

Lemma 1. (see, [41, Theorem 3.1 and Corollary 3.2]) Let $(G, \tau)$ be a semitopological group, $N=\bigcap\{U: e \in U \in \tau\}$ and $K=N \cap N^{-1}$. Then $K$ is a normal subgroup of the group $G$ and $T_{0} G=G / K$ is a $T_{0}$ semitopological group. Moreover, let $\pi: G \rightarrow G / K$ be the quotient homomorphism. Then $U=\pi^{-1} \pi(U)$ for each open set $U \subset G$ and hence the map $\pi$ is clopen.

Lemma 2. A semitopological group $G$ is a paratopological group iff $T_{0} G$ is a paratopological group.

Proof. The sufficiency is evident. The necessity follows from Lemma 1.

Lemma 3. Let $(X, \tau)$ be a weakly semiregular space, $(Y, \sigma)$ be a space and $\pi: X \rightarrow Y$ be a continuous clopen surjection. Then $Y$ is a weakly semiregular space.

Proof. Let $y \in Y$ be any point and $V \in \sigma$ be any open neighborhood of $y$. Pick a point $x \in$ $\pi^{-1}(y)$. Since $\pi^{-1}(V)$ is a neighborhood of $x$ and $X$ is a weakly semiregular space, there exists a regular open neighborhood $U$ of the point $x$, contained in a set $\pi^{-1}(V)$. Then $y=\pi(x) \in$ $\pi(U) \subset \overline{\pi(U)} \subset \pi(\bar{U}) \subset \pi \pi^{-1}(V)=V$ (the third inclusion here holds because the map $\pi$ is closed). Therefore a canonical open set $V^{\prime}=$ int $\overline{\pi(U)}$ is closed and $y \in V^{\prime} \subset \overline{\pi(U)} \subset V$.

Lemma 4. Let $(G, \tau)$ be a weakly semiregular semitopological group. Put $N=\bigcap\{U: e \in U \in$ $\tau$ \}. Then $N$ is a closed normal subgroup of the group $G$ and

$$
N=\bigcap\{\bar{U}: e \in U \in \tau\}=\bigcap\left\{U U^{-1}: e \in U \in \tau\right\}=\bigcap\left\{U^{-1}: e \in U \in \tau\right\} .
$$

Proof. Put $N^{\prime}=\bigcap\{\bar{U}: e \in U \in \tau\}$ and $N^{\prime \prime}=\bigcap\left\{U U^{-1}: e \in U \in \tau\right\}$. The set $N^{\prime}$ is a closed subset of the group $G$. Since for any $V \subset G, \bar{V}=\bigcap\left\{V U^{-1}: e \in U \in \tau\right\}$, we have $N^{\prime}=N^{\prime \prime}$. Moreover, it is easy to see that $N^{-1}=\bigcap\left\{U^{-1}: e \in U \in \tau\right\}, N \subset N^{\prime}, N \subset N^{\prime \prime}$ and $N^{-1} \subset N^{\prime \prime}$. Let $U \in \tau$ be any open neighborhood of the unit of the group $G$ and $x$ be any element of the set $U$. There exists an open neighborhood $V \in \tau$ the of unit of the group $G$ such that $x V \subset U$. Then $x N^{\prime} \subset x \bar{V} \subset \bar{U}$. Since this inclusion holds for an arbitrary element $x$ of the set $U$, we see that $U N^{\prime} \subset \bar{U}$. But $U N^{\prime}$ is an open subset of a group $G$ and hence $N^{\prime} \subset U N^{\prime} \subset \operatorname{int} \bar{U}$. Then $N^{\prime} \subset \bigcap\{\operatorname{int} \bar{U}: e \in U \in \tau\}=\bigcap\{U: e \in U \in \tau\}=N$ (the first equality holds because $G$ is a weakly semiregular space). At last, since $N^{-1} \subset N^{\prime \prime}=N^{\prime} \subset N$, we have the inclusion $N \subset N^{-1}$. 
Let $x, y$ be arbitrary elements of $N$ and $U \in \tau$ be an arbitrary open neighborhood of the unit of the group $G$. Then $x \in N \subset U$. There exists an open neighborhood $V \in \tau$ of the unit of the group $G$ such that $x V \subset U$. Then $y \in N \subset V$. Hence $x y \in x V \subset U$. Since this holds for an arbitrary open neighborhood $U \in \tau$ of the unit of the group $G, x y \in \bigcap\{U: e \in U \in \tau\}=N$. So $N$ is a subsemigroup of the group $G$. Since $N=N^{-1}, N$ is a group.

Let $g$ be an arbitrary element of the group $G$, and $U \in \tau$ be an arbitrary open neighborhood of the unit of the group $G$. There exists an open neighborhood $V \in \tau$ of the unit of the group $G$ such that $g^{-1} V g \subset U$. Then $g^{-1} N g \subset g^{-1} V g \subset U$. Since this holds for an arbitrary open neighborhood $U \in \tau$ of the unit of the group $G, g^{-1} N g \subset \bigcap\{U: e \in U \in \tau\}=N$. So $N$ is a normal subsemigroup of the group $G$.

Proposition 1. Each $T_{0}$ weakly semiregular semitopological group $(G, \tau)$ is semiregular.

Proof. Put $N=\bigcap\{U: e \in U \in \tau\}$. Since $G$ is a $T_{0}$ space, $N \cap N^{-1}=\{e\}$. But by Lemma 4, $N^{-1}=N=\bigcap\left\{U U^{-1}: e \in U \in \tau\right\}=N^{\prime \prime}$. Therefore $N^{\prime \prime}=\{e\}$ and the group $G$ is $T_{2}$.

Lemma 1, Lemma 3 and Proposition 1 imply the following

Proposition 2. If $G$ is a weakly semiregular semitopological group then $T_{0} G$ is a semiregular semitopological group.

We remark that Proposition 2 cannot be generalized for arbitrary quotient groups even of regular paratopological groups, because in [6] Taras Banakh and the author constructed a countable regular abelian paratopological group $G$ containing a closed discrete subgroup $H$ such that the quotient $G / H$ is $T_{2}$ but not $T_{3}$. The group $G / H$ is even not weakly semiregular, because by [31, Proposition 1.5] each weakly semiregular paratopological group is $T_{3}$.

Lemma 5. [35, Theorem 0.5] A $T_{2}$ compact semigroup with separately continouous multiplication and two-sides cancellations is a topological group.

Lemma 6. (see [32, Lemma 5.4], [41, Proposition 3.2], or [8, Proposition 3.2]) Each compact paratopological group is a topological group.

Theorem 1. Each weakly semiregular compact semitopological group $G$ is a topological group.

Proof. By Proposition 2, $T_{0} G$ is a semiregular compact semitopological group. By Lemma 5, $T_{0} G$ is a topological group. By Lemma $2, G$ is a paratopological group. By Lemma $6, G$ is a topological group.

Let us illustrate the topic by the following simple

Proposition 3. Let $G$ be a group endowed with the cofinite topology, that is a set $U \subset G$ is open in $G$ iff $U=\varnothing$ or a set $G \backslash U$ is finite. Then $G$ is a $T_{1}$ semitopological group and the following conditions are equivalent.

1. The group $G$ is a paratopological group.

2.1. The group $G$ is $T_{2}$.

2.2. The group $G$ is weakly semiregular. 


\subsection{The group $G$ is quasiregular.}

3. The group $G$ is finite.

Proof. The continuity of shifts on the group $G$ and implications $3 \Rightarrow *$ are obvious, implications 2.* $\Rightarrow 3$ follows from the fact that if the group $G$ is infinite then each nonempty open subset of $G$ is dense in $G$. It remains to show an implication $1 \Rightarrow 3$. Suppose to the contrary that $G$ is an infinite paratopological group. Pick an element $x \in G \backslash\{e\}$. Since the multiplication at the unit of $G$ is continuous, there exists a finite set $F \subset G \backslash\{e\}$ such that $(G \backslash F)^{2} \subset G \backslash\{x\}$. Since the group $G$ is infinite, there exists a point $y \in G \backslash\left(F \cup x F^{-1}\right)$. Then $y(G \backslash F) \ni x$, a contradiction.

Example 2. There exists a $T_{1}$ quasiregular compact quasitopological group $G$, which is not a paratopological group. Let $G=\mathbb{T}=\{z \in \mathbb{C}:|z|=1\}$ be the unit circle. We define an open base $\mathcal{B}$ at the unit of a topology of a semitopological group on $G$ by putting $\mathcal{B}=\left\{U_{n}: 0<n \in\right.$ $\mathbb{Z}\}$, where $U_{n}=\{z \in \mathbb{C} \backslash\{(-1,0)\}: \arg z \in(-1 / n, 1 / n) \cup(\pi-1 / n, \pi+1 / n)\}$.

Example 3. There exists a $T_{2}$ quasiregular sequentially compact quasitopological group $G$, which is not a paratopological group. Let

$$
G=\Sigma_{\omega_{1}} \mathbb{Z}_{2}=\left\{x \in \mathbb{Z}_{2}^{\omega_{1}}:\left|\left\{\alpha: x_{\alpha} \neq 0\right\}\right| \leq \omega\right\} .
$$

Put $\mathcal{B}=\left\{U_{A} \backslash S: A\right.$ is a finite subset of $\left.\omega_{1}\right\}$, where

$$
U_{A}=\left\{x \in G: x_{\alpha}=x_{\beta} \text { for each } \alpha, \beta \in A\right\}
$$

and

$$
S=\left\{x \in G: x_{0}=1 \text { and } x_{\gamma} \geq x_{\delta} \text { for each } \gamma<\delta<\omega_{1}\right\} .
$$

We claim that the family $\mathcal{B}$ satisfies Pontrjagin conditions (see [30, Proposition 1]). Indeed, the one non-evident of these conditions for the family $\mathcal{B}$ is: for each $U \in \mathcal{B}$ and for each point $x \in U$ there exists $U^{\prime} \in \mathcal{B}$ such that $x+U^{\prime} \subset U$. Let's check this. Let $\mathcal{B} \ni U=U_{A} \backslash S$, where $A$ is a finite subset of $\omega_{1}$ and $x \in U$. If $x=0$ then it suffices to put $U^{\prime}=U$. If $x \neq 0$ then there exists an index $\gamma^{\prime} \in \omega_{1}$ such that $x_{\gamma^{\prime}}=1$. Since $x \in \Sigma_{\omega_{1}} \mathbb{Z}_{2}$, there exists an index $\gamma^{\prime}<\delta^{\prime}<\omega_{1}$ such that $x_{\delta^{\prime}}=0$. Since $x \notin S$, there exist indexes $\gamma^{\prime \prime}, \delta^{\prime \prime} \in \omega_{1}, \gamma^{\prime \prime}<\delta^{\prime \prime}$ such that $x_{\gamma^{\prime \prime}}=0$ and $x_{\delta^{\prime \prime}}=1$. Put $A^{\prime}=A \cup\left\{\gamma^{\prime}, \gamma^{\prime \prime}, \delta^{\prime}, \delta^{\prime \prime}\right\}$ and $U^{\prime}=U_{A^{\prime}}$. Then $x+U^{\prime} \subset U$. Hence the family $\mathcal{B}$ is an open base at the unit of a topology of a semitopological group on $G$. Denote this topology as $\tau$. Since $U_{A^{\prime}} \supset \overline{U_{A^{\prime}} \backslash S}$, the group $(G, \tau)$ is quasiregular. Since the set $U_{A}$ is a group for any subset $A$ of $\omega_{1}$ and $\cap\left\{U_{A}: A\right.$ is a finite subset of $\left.\omega_{1}\right\}=\{0\}$, the group $(G, \tau)$ is $T_{2}$. Since the topology $\tau$ is weaker than the sequentially compact topology on the set $\Sigma_{\omega_{1}} \mathbb{Z}_{2}$, induced from the Tychonoff product, the group $(G, \tau)$ is sequentially compact too. At last, to show that $(G, \tau)$ is not a paratopological group, it suffices to show that for any finite set $A \subset \omega_{1}$ there exist points $x, y \in U_{A} \backslash S$ such that $x+y \in S$. Fix arbitrary two indexes $\alpha, \beta \in \omega_{1}$ such that $\sup A<\alpha<\beta$. For each $\gamma \in \omega_{1}$ put $x_{\gamma}=1$ if $\gamma \in\{\alpha, \beta\}$ and $x_{\gamma}=0$ otherwise. For each $\gamma \in \omega_{1}$ put $y_{\gamma}=1$ if $\alpha \neq \gamma \leq \beta$ and $y_{\gamma}=0$ otherwise.

Recall that a topological group $G$ is precompact if for each neighborhood $U$ of the unit of $G$ there exists a finite subset $F$ of $G$ such that $F U=G$ (or, equivalently $U F=G$ ). 
Theorem 2. Let $(G, \sigma)$ be a $T_{2}$ precompact topological group, $(G, \tau)$ be a weakly semiregular semitopological group and $\tau \subset \sigma$. Then $(G, \tau)$ is a topological group.

Proof. Let $(\hat{G}, \hat{\sigma})$ be a Rarkov completion of the group $(G, \sigma)$. Since the group $G$ is a dense precompact subset of the group $(\hat{G}, \hat{\sigma})$, by Corollary 3.7.6 from [5], the group $(\hat{G}, \hat{\sigma})$ is precompact. Since the group $(\hat{G}, \hat{\sigma})$ is Ray̌kov complete, by Theorem 3.7.15 from [5] it is compact.

In this proof as ${ }^{-}$we denote the closure with respect to the topology $\hat{\sigma}$.

Put $N=\bigcap\{\bar{U}: e \in U \in \tau\}$. We claim that $N$ is a normal subgroup of the $\operatorname{group}(\hat{G}, \hat{\sigma})$. Indeed, let $x, y$ be any elements of $N, U \in \tau$ be an any open neighborhood of the unit of the group $G$, and $\hat{W}=(\hat{W})^{-1} \in \hat{\sigma}$ be any symmetric open neighborhood of the unit of the group $\hat{G}$. Then there exists an element $u \in U \cap \hat{W} x$. There exists an open neighborhood $V \in \tau$ of the unit of the group $G$ such that $u V \subset U$. Then there exists an element $v \in V \cap y \hat{W}$. Then $x y \in \hat{W} u v \hat{W} \subset \hat{W} U \hat{W}$. Since this holds for any symmetric open neighborhood $\hat{W}=(\hat{W})^{-1} \in$ $\hat{\sigma}$ of the unit of the group $\hat{G}, x y \in \bar{U}$. Since this holds for any open neighborhood $U \in \tau$ of the unit of the group $G, x y \in \bigcap\{\bar{U}: e \in U \in \tau\}=N$. So $N$ is a closed subsemigroup of a $T_{2}$ compact topological group $(\hat{G}, \hat{\sigma})$. By Lemma $5, N$ is a group. Let $g$ be any element of the group $G$ and $U \in \tau$ be any open neighborhood of the unit of the group $G$. Since $(G, \tau)$ is a semitopological group and $g^{-1} e g=e$ there exists an open neighborhood $V \in \tau$ of the unit of the group $G$ such that $g^{-1} V g \subset U$. By continuity of multiplication on the group $(\hat{G}, \hat{\sigma})$, $g^{-1} N g \subset g^{-1} \bar{V} g \subset \bar{U}$. Since this holds for any open neighborhood $U \in \tau$ of the unit of the group $G, g^{-1} N g \subset \bigcap\{\bar{U}: e \in U \in \tau\}=N$. Now suppose that there exists an element $\hat{g}$ of the group $\hat{G}$ such that $(\hat{g})^{-1} N \hat{g} \not \subset N$. Then there exists an element $x \in N$ such that $(\hat{g})^{-1} x \hat{g} \notin N$. Since $N$ is a closed subset of the group $(\hat{G}, \hat{\sigma})$ and the multiplication on the group $(\hat{G}, \hat{\sigma})$ is continuous, there exists a symmetric open neighborhood $\hat{W}=(\hat{W})^{-1} \in \hat{\sigma}$ of the unit of the group $\hat{G}$ such that $\hat{W}(\hat{g})^{-1} x \hat{g} \hat{W} \cap N=\varnothing$. Since the group $(G, \sigma)$ is dense in its completion $(\hat{G}, \hat{\sigma})$, there exists an element $g \in G \cap \hat{g} \hat{W}$. But then $g^{-1} x g \in \hat{W}(\hat{g})^{-1} x \hat{g} \hat{W} \notin N$, a contradiction. Therefore $(\hat{g})^{-1} N \hat{g} \subset N$ for each element $(\hat{g}) \in \hat{G}$. Thus $N$ is a normal subgroup of the group $\hat{G}$.

Define a topology $\hat{\sigma}_{N}$ on the group $\hat{G}$ by putting $\hat{\sigma}_{N}=\{\hat{W} N: \hat{W} \in \hat{\sigma}\}$. It is easy to check that $\left(\hat{G}, \hat{\sigma}_{N}\right)$ is a topological group. We claim that $\hat{\sigma}_{N} \mid G=\tau$. Let's check this.

$\left(\hat{\sigma}_{N} \mid G \subset \tau\right)$ Let $\hat{W} \in \hat{\sigma}$ be any non-empty set and $x \in \hat{W} N \cap G$ be any point. Then $e \in$ $x^{-1} \hat{W} N$, so $\bigcap\{\bar{U}: e \in U \in \tau\}=N \subset x^{-1} \hat{W} N$. Since $x^{-1} \hat{W} N$ is an open subset of the compact group $\left(\hat{G}, \hat{\sigma}_{N}\right)$, there exists a set $e \in U \in \tau$ such that $\bar{U} \subset x^{-1} \hat{W} N$. Then $x U$ is a neighborhood of the point $x$ in the topology $\tau$ and $x U \subset \hat{W} N \cap G$.

$\left(\hat{\sigma}_{N} \mid G \supset \tau\right)$ Let $U \in \tau$ be any open neighborhood of the unit of the group $G$. We claim that $\bar{U} N \subset \bar{U}$. Indeed, let $x$ be any element of the set $U$. There exists an open neighborhood of $V \in \tau$ the unit of the group $G$ such that $x V \subset U$. Then $x N \subset x \bar{V} \subset \bar{U}$. Since this inclusion holds for any element $x$ of the set $U$, we see that $U N \subset \bar{U}$. Let $y$ be any element of the set $N$. Then $U y \subset \bar{U}$ and $U \subset \bar{U} y^{-1}$. Since the set $\bar{U} y^{-1}$ is closed in the group $\left(\hat{G}, \hat{\sigma}_{N}\right)$, we see that $\bar{U} \subset \bar{U} y^{-1}$. At last, since this inclusion holds for any element $y$ of the set $N$, we see that $\bar{U} N \subset \bar{U}$. Since $\hat{\sigma} \mid G \supset \tau$, there exists an open neighborhood $\hat{W} \in \hat{\sigma}$ of the unit of the group $G$ such that $\hat{W} \cap G \subset U$. Since the set $G$ is dense in the space $(\hat{G}, \hat{\sigma}), \hat{W} \subset \overline{\hat{W} \cap G} \subset \bar{U}$. Then $\hat{W} N \subset \bar{U} N \subset \bar{U}$. But $\hat{W} N \cap G \in \tau$, because $\hat{\sigma}_{N} \mid G \subset \tau$. Therefore $\hat{W} N \cap G \subset \operatorname{int}_{\tau}(\bar{U} \cap G) \subset$ $\operatorname{int}_{\tau} \bar{U}^{\tau}$ (we have $\bar{U} \cap G \subset \bar{U}^{\tau}$, because $\hat{\sigma} \mid G \supset \tau$ ). At last, since $U \in \tau$ is any open neighborhood of the unit of the weakly semiregular group $G$, we have that $\left(\hat{\sigma}_{N} \mid G \supset \tau\right)$. 
Thus, since $\hat{\sigma}_{N} \mid G=\tau,(G, \tau)$ is a topological group.

Theorem 3. Let $(G, \sigma)$ be a $T_{2}$ feebly compact paratopological group, $(G, \tau)$ be a $T_{3}$ semitopological group and $\tau \subset \sigma$. Then $(G, \tau)$ is a topological group.

Proof. The group $G$ endowed with the topology $\sigma_{s r}$ is a feebly compact $T_{2}$ and $T_{3}$ paratopological group. By [8, Proposition 3.15], $\left(G, \sigma_{s r}\right)$ is a feebly compact topological group. Hence the group $\left(G, \sigma_{s r}\right)$ is precompact. Let $U \in \tau$ be an arbitrary set and $x \in U$ be an arbitrary point. Since topology $\tau$ is $T_{3}$, there exists an open neighborhood $V \in \tau$ of the point $x$ such that $\bar{V}^{\tau} \subset U$. Since $\tau \subset \sigma, V \in \sigma$. Then $x \in V=\operatorname{int}_{\tau} V \subset \operatorname{int}_{\tau} \bar{V}^{\sigma} \subset \operatorname{int}_{\sigma} \bar{V}^{\sigma} \subset \operatorname{int}_{\sigma} \bar{V}^{\tau} \subset \bar{V}^{\tau} \subset U$. Since $\operatorname{int}_{\sigma} \bar{V}^{\sigma} \in \sigma_{s r}, \tau \subset \sigma_{s r}$, and $\left(G, \sigma_{s r}\right)$ is a weakly semiregular space, by Theorem $2,(G, \tau)$ is a topological group.

\section{ACKNOWLEDGEMENTS}

The author is grateful to Warren Moors for providing him the article [27].

\section{REFERENCES}

[1] Alas O.T., Sanchis M. Countably Compact Paratopological Groups. Semigroup Forum 2007, 74, $423-438$. doi:10.1007/s00233-006-0637-y

[2] Arhangel'ski1 A.V., Choban M.M. On paratopological groups and pseudocompactness. Preprint.

[3] Arhangel'skiř A.V., Choban M.M., Kenderov P.S. Topological games and topologies on groups. Math. Maced. 2010, 8, 1-19.

[4] Arhangel'skiĭ A.V., Reznichenko E.A. Paratopological and semitopological groups versus topological groups. Topology Appl. 2005, 151, 107-119. doi:10.1016/j.topol.2003.08.035

[5] Arhangel'ski1 A.V., Tkachenko M. Topological groups and related structures. Atlantis Press, Paris; World World Sci. Publ., Hackensack, NJ, 2008.

[6] Banakh T.O., Ravsky A.V. The regularity of quotient paratopological groups. Mat. Stud. 2018, 49 (2), $144-149$. doi:10.15330/ms.49.2.144-149

[7] Banakh T.O., Ravsky A.V. Each regular paratopological group is completely regular. Proc. Amer. Math. Soc. 2017, 145 (3), 1373-1382. doi:10.1090/proc/13318

[8] Banakh T.O., Ravsky A.V. Feebly compact paratopological groups (version 7). -arXiv:1003.5343v7

[9] Bouziad A. Every Čech-analytic Baire semitopological group is a topological group. Proc. Amer. Math. Soc. 1996, 124 (3), 953-959.

[10] Brand N. Another note on the continuity of the inverse. Arch. Math. (Basel) 1982, 39, 241-245. doi:10.1007/BF01899530

[11] Dikranjan D.N., Prodanov I.R., Stoyanov L.N. Topological Groups: Characters Dualities and Minimal Group Topologies, (2nd edn.), In: Kabayashi S., Hewitt E. (Eds.) Monographs and Textbooks in Pure and Applied Mathematics, 130. Marcel Dekker, New York 1989.

[12] Dorantes-Aldama A., Shakhmatov D., Selective sequential pseudocompactness. Topology Appl. 2017, 222 , 53-69. doi:10.1016/j.topol.2017.02.016

[13] van Douwen E.K., Reed G.M., Roscoe A.W., Tree I.J. Star covering properties. Topology Appl. 1991, 39 (1), 71-103. doi:10.1016/0166-8641(91)90077-Y

[14] Ellis R. Locally compact transformation groups. Duke Math. J. 1957, 24, 119-125. doi:10.1215/S0012-7094-5702417-1 
[15] Ellis R. A note on the continuity of the inverse. Proc. Amer. Math. Soc. 1957, 8, 372-373. doi:10.1090/S0002-99391957-0083681-9

[16] Engelking R. General topology. Heldermann, Berlin, 1989.

[17] Gutik O.V., Ravsky A.V. On old and new classes of feebly compact spaces. Visnyk of the Lviv Univ. Ser. Mech. Math. 2018, 85, 48-59. doi:10.30970/vmm.2018.85.048-059

[18] Katĕtov M. On H-closed extensions of topological spaces. C̆asopis Pĕst. Mat. Fys. 1947, 72, 17-32.

[19] Kenderov P.S., Kortezov I.S., Moors W.B. Topological games and topological groups. Topology Appl. 2001, 109, 157-165. doi:10.1016/S0166-8641(99)00152-2

[20] Korovin A. Continuous actions of Abelian groups and topological properties in $C_{p}$-theory. Ph.D. Thesis, Moscow State University, Moscow, 1990. (in Russian)

[21] Korovin A. Continuous actions of pseudocompact groups and the topological group axioms. Deposited in VINITI 1990, \#3734-D, Moscow. (in Russian)

[22] Li P., Tu J.-J., Xie L.-H. Notes on (regular) T3-reflections in the category of semitopological groups. Topology Appl. 2014, 178, 46-55. doi:10.1016/j.topol.2014.09.001

[23] Lipparini P. A very general covering property. -arXiv:1105.4342

[24] Lawson J.D. Joint continuity in semitopological semigroups. Illinois J. Math. 1974, 18 (2), $275-285$.

[25] Matveev M. A survey on star covering properties. Topology Atlas. Preprint \#330. http://at.yorku.ca/v/a/a/a/19.htm

[26] Montgomery D. Continuity in topological groups. Bull. Amer. Math. Soc. 1936, 42, 879-882. doi:10.1090/S00029904-1936-06456-6

[27] Moors W.B. Some Baire semitopological groups that are topological groups. Topology Appl. 2017, 230, $381-392$. doi:10.1016/j.topol.2017.08.042

[28] Pfister H. Continuity of the inverse. Proc. Amer. Math. Soc 1985, 95, 312-314. doi:10.1090/S0002-9939-19850801345-5

[29] Pontrjagin L.S. Continuous groups. Nauka, Moscow, 1973. (in Russian)

[30] Ravsky A.V. Paratopological groups I. Mat. Stud. 2001, 16 (1), 37-48.

[31] Ravsky A.V. Paratopological groups II. Mat. Stud. 2002, 17 (1), 93-101.

[32] Ravsky A.V. The topological and algebraical properties of paratopological groups. Ph.D. Thesis Lviv University, Lviv, 2002. (in Ukrainian)

[33] Ravsky A.V. Post \#209491 at MathOverflow,

[34] Ravsky A.V., Reznichenko E.A. The continuity of inverse in groups. In: Zagorodnuyk A.V., Hryniv R.O. Book of Abstracts of International Conference on Functional Analysis and its Applications Dedicated to the 110th anniversary of Stefan Banach, Lviv, Ukraine, May 28-31, 2002, Publ. Cent. of Ivan Franko National University of Lviv, Lviv, 2002, 170-172.

[35] Reznichenko E.A. Extension of functions defined on products of pseudocompact spaces and continuity of the inverse in pseudocompact groups. Topology Appl. 1994, 59, 33-44. doi:10.1016/0166-8641(94)90021-3

[36] Reznichenko E.A. Čech complete semitopological group are topological groups. (Preprint).

[37] Stephenson Jr. R.M. Initially $\kappa$-compact and related compact spaces. In K. Kunen, J. E. Vaughan (Eds.), Handbook of Set-Theoretic Topology, Elsevier Science Publishers B.V., Amsterdam, 1984, 603-632. doi:10.1016/B978-0444-86580-9.50016-1

[38] Stone M.H. Applications of the theory of Boolean rings to general topology. Trans. Amer. Math. Soc. 1937, 41, 375-481. doi:10.1090/S0002-9947-1937-1501905-7

[39] Seminar "Topology \& its Applications" at Chair of Geometry and Topology, Mechanics and Mathematics Faculty, Ivan Franko National University of Lviv. 28 November 2016. Not published. 
[40] Tkachenko M. Semitopological and paratopological groups vs topological groups. In: Hart K.P., van Mill J., Simon P. (Eds.), Recent Progress in General Topology III, Springer Science \& Business Media, 2013, 803-859.

[41] Tkachenko M. Axioms of separation in semitopological groups and related functors. Topology Appl. 2014, 161, 364-376. doi:10.1016/j.topol.2013.10.037

[42] Vaughan J.E. Countably compact and sequentially compact spaces. In K. Kunen, J. E. Vaughan (Eds.), Handbook of Set-Theoretic Topology, Elsevier Science Publishers B.V., Amsterdam, 1984, 569-602. doi:10.1016/B978-0444-86580-9.50015-X

[43] Wallace A.D. The structure of topological semigroups. Bull. Amer. Math. Soc. 61 (1955), 95-112.

Received 17.08.2019

Равський О.В. Аещу про компактоподібні напівтопологічні групи // Карпатські матем. публ. 2019. — T.11, №2. - С. 442-452.

Отримано деякі результати пов'язані з аксіомами відокремлення та автоматичною неперервністю у компактоподібних напівтопологічних групах. Зокрема, наведена напіврегулярна напівтопологічна група $G$, котра не $\epsilon T_{3}$. Показано, шо кожна слабко напіврегулярна компактна напівтопологічна група $є$ топологічною групою. 3 іншого боку, побудовані приклади квазірегулярних $T_{1}$ компактної та $T_{2}$ секвенціально компактної квазітопологічних груп, котрі не $є$ паратопологічними групами. Також показано, що напівтопологічна група $(G, \tau) \in$ топологічною групою за умови існування такої гаусдорфової топології $\sigma \supset \tau$ на $G$, що $(G, \sigma) \epsilon$ прекомпактною топологічною групою і $(G, \tau) \in$ слабко напіврегулярною або $(G, \sigma) \in$ слабко компактною паратопологічною групою і $(G, \tau) \in T_{3}$.

Ключові слова $і$ фрази: напівтопологічна група, паратопологічна група, компактоподібна напівтопологічна група, компактоподібна паратопологічна група, неперервність оберненого, сукупна неперервність, аксіоми відокремлення, зліченно-компактна паратопологічна група, слабко компактна паратопологічна група, зліченно-компактна топологічна група. 